sult, besides being of service in the study of the uniform convergence of Fourier's series, furnishes a means for determining an upper limit to the number of terms necessary to take in a given Fourier development in order that the error committed by stopping with the last term taken shall be less than some assigned quantity, this being true when the series is considered for all values of $x$ lying within the interval $(a, b)$ ( $a$ and $b$ included), it being assumed throughout that $f(x)$ satisfies certain preliminary conditions (such as Dirichlet's conditions) at every point of this interval.

Columbia University.

F. N. CoLE.

\title{
THE JANUARY MEETING OF THE CHICAGO SECTION.
}

THE tenth regular meeting of the Chicago Section of the American Mathematical Society was held at Northwestern University, Evanston, Illinois, on Thursday and Friday, January 2-3, 1902. Two sessions were held each day, opening at 10 o'clock A. M. and 2.30 o'clock P. M. In the absence of Professor E. H. Moore, President of the Society, during the morning sessions, Professor Ziwet occupied the chair on the first day and Professor White on the second day. The attendance at the various sessions was unusually large, including a number of prominent educators in the vicinity of Chicago, and the following twenty-five members of the Society :

Professor R. J. Aley, Dr. G. A. Bliss, Professor Oskar Bolza, Dr. C. L. Bouton, Professor D. F. Campbell, Mr. A. Crathorne, Professor E. W. Davis, Professor L. E. Dickson, Professor L. W. Dowling, Dr. J. W. Glover, Dr. T. P. Hall, Professor Thomas F. Holgate, Dr. H. G. Keppel, Dr. Kurt Laves, Professor H. Maschke, Professor E. H. Moore, Dr. F. R. Moulton, Miss I. M. Schottenfels, Professor G. T. Sellew, Professor J. B. Shaw, Professor E. J. Townsend, Professor C. A. Waldo, Dr. Jacob Westlund, Professor H. S. White, Professor Alexander Ziwet.

At the business session on Friday morning the following officers of the section were elected for the ensuing year : Secretary. Professor Thomas F. Holgate ; Additional members of the programme committee, Profeswor E. J. Townsend and Professor L. W. Dowling.

The following papers were presented and read : 
(1) Professor M. W. Haskell : "A fundamental theorem in the geometry of the tetrahedron."

(2) Professor M. W. HAskell: "A theorem for the twisted cubic analogous to Pascal's theorem."'

(3) Professor M. W. Haskell : "A special cubic transformation in space."

(4) Professor H. S. White : "Note on a twisted curve connected wilh an involution of pairs of points in a plane."

(5) Dr. J. W. Glover : "On the derivation of the asymptotes of an algebraic curve from the definition" (preliminary communication).

(6) Professor Arnold EMch : "Algebraic transformations of a complex variable realized by linkages."

(7) Professor L. W. Dowling: "On the conformal representation of the isosceles triangle containing an angle of 120 degrees."

(8) Professor E. H. Moone : "On Hilbert's plane arguesian geometry."

(9) Dr. F. R. Moulton: "A simple non-arguesian geometry."

(10) Dr. JACOB Westlund : "Note on multiply perfect numbers."

(11) Dr. JACOB Westlund : "On the class number of a particular cyclotomic number field."

(12) Dr. C. L. Bouton : "The equivalence of linear differential equations for a transformation of the independent variable."

(13) Dr. T. P. HALL : "An algebra of space."

(14) Professor J. B. SHAw : "Commutativity of matrices and application to the theory of linear associative algebra."

(15) Dr. H. G. KePpel: "A cubic three-way locus in four-fold space."

(16) Dr. J. C. Fields : "An equivalent of Plücker's formulæ.'

(17) Professor H. B. Newson : "On the product of linear substitutions."

(18) Professor G. A. Miller : "On the groups of order $p^{m}$ which contain operators of order $p^{m-2}$."

(19) Professor L. E. Dickson : "Some simplifications in the theory of linear groups."

In the absence of the authors, Dr. Fields's paper and Professor Haskell's third paper were read by Professor White. Professor Emch's and Professor Miller's papers by Professor Moore, Professor Newron's paper by Professor Dowling, and Profe'ssor Haskell's first and second papers by the Secretary ; Professor Dickson's paper was read by title. 
In addition to the papers announced on the regular programme, Professor Townsend opened a discussion on the subject of the best preparation in the undergraduate course for post-graduate study in mathemalics, which developed into a discussion of the programme leading to the master's degree. The question of migration from one college or university to another was considered, and a committee consisting of Professors Waldo, Townsend, and Bolza was appointed to consider and report a scheme of equivalent requirements for candidates proceeding to their second degree with mathematics as their major subject.

An interesting feature of the meeting was a dinner served on the evening of Thursday, January $2 d$, in one of the University buildings, and participated in by twenty-six persons. This was followed by an exhibit by Dr. Keppel of about fifty portraits of eminent mathematicians.

Abstracts of the papers presented are as follows :

Professor Haskell's first paper contains the following theorem with some special cases: If six points are chosen at random on the six edges of a tetrahedron, one point on each edge, the four spheres determined by a vert. $x$ of the tetrahedron and the three points chosen on the edges meeting in that vertex, have one point in common. His second paper gives an analytical proof of the following theorem and its reciprocal : If eight points $A, B, C, D, E, F, G . H$, are chosen at random on a twisted cubic, and the eight planes $A B C, B C D, \cdots, H A B$, are considered, then the four rays of intersection of pairs of opposite planes, $A B C-E F G$, $B C D-F G H, C D E-G H A, D E F-H A B$. are generators of the same set of a ruled hyperboloid. His third paper contains a discussion of some of the interesting properties of the birational transformation which expresses the relation between a point and its harmonic conjugate with respect to a twisted cubic.

All involutions of pairs of points in a plane are divided by Bertini into four classes, distinct under the group of all Cremona transformations. The simplest of these is the relation of perspective homography. To determine this, two pairs of points are sufficient. When two pairs in such an involution are given, any third pair must satisfy two conditions. Hence if three pairs of points are given in arbitrary situation in space, a center from which they shall be projected by rays in an involution of this simplest type is subjected to two conditions, and must describe a twisted curve. In Pro- 
fessor White's paper this curve is investigated, and found to be of order 5 and deficiency 0 .

Dr. Glover's preliminary communication takes up the general problem of the derivation of the asymptotes of the algebraic curve $u(x, y)=0$ by forming the equation of the tangent at the point $(x, y)$ and then expressing the intercept and angle as fractions whose limits are investigated as the point of contact is indefinitely removed from the origin. This method leads to the usual equations for determining the values of the constants fixing the asymptotes.

In a paper appearing in 1895 in the Comptes rendus and also later in his book on Kinematics, Koenigs proved that the general system of (real) algebraic relations between any finite number of (real) points in space could be generated by a space linkage, that is, a linkage in which various rigid pieces are connected together at spherical pivots. Professor Emch remarks that of course this covers the case of any system of (real) algebraic relations between a finite number of (real) points which are coplanar, and further, by the usual analytical considerations, the case of any system of algebraic relations between $n$ points in the complex plane.

The single algebraic relation between two complex points may obviously be obtained by proper combination of linkages capable of effecting the determination from two arbitrary points $Z_{1}$ and $Z_{2}$, of the sum $Z_{3}=Z_{1}+Z_{2}$ on the one hand, and on the other of the product $Z_{4}=Z_{1} Z_{2}$. Professor Emch has constructed a plane linkage for the first of these two relations and makes use of a plane linkage constructed by Kleiber for the second relation. In the plane linkage the pivots are of the nature of pins perpendicular to the plane in which the linkage is operating.

Professor Dowling presented the results of a study of the integral which gives the conformal representation of the particular triangle mentioned in the title of his paper, and of the Riemann surface upon which it is an abelian integral of the first kind. The situation of the triangle corresponding to the positive half of the upper sheet is assumed and the position of the triangles corresponding to the remaining half sheets is found. The abelian integral being of deficiency two, a similar study is made of the associated independent abelian integral, and the reduction to an elliptic integral is discussed. 
In his Gauss-Weber Festschrift : "Grundlagen der Geometrie," Hilbert devotes Chapter V to Desargues's theorem, and shows (page 70) that a plane geometry (I, II, III), that is, one in which the plane axioms of connection and order and the parallel axiom hold, is embodied in a spatial geometry (I, II, III) if and only if the theorem of Desargues holds in the plane geometry. In proof of the sufficiency of this condition, Hilbert develops in a plane geometry, (I, II, III, $D$ ), in which Desargues's theorem $D$ does hold, an algebra of (directed) segments $O A$ lying on a fixed line $l$, and on that line having a fixed extremity $O$; and on the basis of this algebra he constructs analytically the three-dimensional space (I, II, III) which contains the initial plane (I, II, III, $D$ ). In this algebra of segments, while the definitions are simple, the proofs of the correctness of some of the rules of calculation are unduly complicated. These proofs and the whole theory of the algebra Professor Moore has simplified and enriched by the introduction of a vector analysis in the plane, which embodies the segmental algebra of Hilbert as to its relations of addition, and by a suitable use of the non-essential auxiliary elements of the Hilbert definitions.

In Chapter V of Professor Hilbert's memoir on the foundations of geometry, he arrives at the following important theorem: The necessary and sufficient conditions that a plane geometry fulfilling the projective axioms of plane geometry may be a part of (or set in) a spatial geometry of more than two dimensions fulfilling the projective axioms, is that Desargues's theorem shall be fulfilled. In order to prove that the fulfillment of Desargues's theorem is necessary, Hilbert remarked the well known fact that the theorem can be proved from a spatial geometry of more than two dimensions fulfilling the axioms in question. He then exhilited a non-arguesian geometry fulfilling the plane axioms in question. His example is somewhat complicated, involving the intersections of an ellipse and a system of circles. In short, it depends upon the solution of simultaneous quadratic equations. Dr. Moulton's paper proves this part of the theorem by the exhibition of an extremely simple geometry fulfilling the axioms in question, and in which Desargues's theorem is not true. The demonstrations involve only linear equations. The geometry consists of ordinary euclidean points in a plane, but the lines are described in terms of the euclidean lines as follows:

The lines 


$$
x-a=\delta_{y, \theta} \tan \theta y,
$$

where $\delta_{y, \theta}=1$ if $y<0$ or if $y>0$ and $\theta<0$, and $\delta_{y \theta}=2$ if $y>0$, and $\theta>0$, are to be considered as the straight lines in the new geometry. It is easily seen that they fulfill the projective plane axioms, and that in this geometry Desargues's theorem is not true.

In a paper presented at the meeting of the Chicago section in April last, and published in the Annals of Mathematics, series 2, volume 2, Dr. Westlund determined all multiple perfect numbers of multiplicity 3 of the form $m=p_{1}^{a_{1}} p_{2}^{a_{3}} p_{3}$. In the present paper all numbers of multiplicity 3 of the form $m=p_{1}{ }^{a_{1}} p_{2} p_{3} p_{4}$, where $p_{1}, p_{2}, p_{3}, p_{4}$ are distinct primes, are determined.

In Dr. Westlund's second paper it is shown how the first factor of the class number of the cyclotomic number-field $k\left(e^{2 \pi i / s^{\kappa}}\right)$ can be determined. The results are then applied to the particular cases $x=2,3$.

Two linear differential equations are said to be equivalent for change of the independent variable when it is possible to transform the first into the second by a change of the independent variable alone. Dr. Bouton's paper gives the necessary and sufficient conditions for this equivalence in invariant form in the case of equations of the $n$th order. The condition for the general equation of the second order $y^{\prime \prime}+2 p_{1}(x) y^{\prime}+p_{2}(x) y=0$, is found as follows: Compute the invariants

$$
I_{1}=\frac{\left(p_{2}^{\prime}+4 p_{1} p_{2}\right)^{2}}{p_{2}{ }^{3}}, \quad I_{2}=\frac{p_{2}^{\prime \prime}+4 p_{1}^{\prime} p_{2}+10 p_{1} p_{2}^{\prime}+24 p_{1}^{2} p_{2}}{p_{2}{ }^{2}} .
$$

For a given equation these are in general functions of $x$, so that $x$ may be eliminated, giving $I_{2} \equiv \psi\left(I_{1}\right)$. The same thing can be done for the second given differential equation. The neces-ary and sufficient condition for the equivalence of the two differential equations is that the two functions $\psi$ so determined shall be the same. Various special cases require separate consideration. For the equation of the $n$th order there are $n-1$ such functions $\psi$.

In Dr. Hall's paper, a vector algebra is sought in which each algebraic expression represents a geometric configuration, and each algebraic operation a motion in space. 
From the laws of motion the laws of algebraic operations are derived, and the resulting algebra is found to include quaternions as a special form. In two fold space it leads to the plane complex number and in all hyperspace it has a quaternion-like rotor.

It is shown in Professor Shaw's paper that, by a very simple form of the square representation of a matrix, the condition that $\varphi_{j}$ shall satisfy the equation

$$
\varphi_{j} \varphi_{i}=\varphi_{i} \varphi_{j}
$$

can be easily found graphically. The totality of matrices on the same ground commutative with $\varphi_{i}$ is determined, and also the relations of the various independent components of the most general commutative matrix. The two classes of components are considered.

In the application, the use of the preceding in determining linear associative algebras is developed, and the full structure of any such algebra exhibited. The matrix explanation of the Lie-Scheffers theory of multiple algebras is brought out, and the real basis of the classification into quaternion and non-quaternion forms is shown. The place of Peirce's study is also shown. Certain developments of the theory in extension of it are also presented.

In Dr. Keppel's paper the cubic three-spread containing a double plane in four-fold space is considered. This locus has a single infinity of plane generators, these generators meeting each other in the points of the double plane while consecutive plane generators intersect in the points of a conic in this double plane. A section of this locus by a three-flat is a ruled cubic surface, the section of the double plane being the double line. The ruled cubic surface will have two real distinct, two imaginary, or two coincident pinch points according as this line meets the conic in two real distinct, in two imaginary, or in two coincident points. Seven different ways of generating the locus geometrically are found, and sections of these by a three-flat give all the well known different ways of generating the ruled cubic surface in three-fold space. One way of generating the locus, namely, by means of two plane point fields collinearly related, gives the locus as the envelope of its double infinity of tangent three-flats, and this method provides a simple representation of the locus on the plane. The reciprocal of the locus is the general cubic three-spread in four-fold space, 
and systems of these cubic surfaces lie on the cubic threespread here considered.

Dr. Fields's paper may be characterized as follows: If $F(z, u)=0$ be the equation to an algebraic curve of degree $n$ in $u$, the $n$ branches corresponding to a value $z=a$ will group themselves in a certain number $r_{k}$ of cycles of orders $v_{1}^{(\kappa)}, \cdots, v_{r_{\kappa}}{ }^{(\kappa)}$. The lowest exponents in the developments of the function $F_{u}^{\prime}(z, u)$ corresponding to these cycles we shall indicate by $m_{1}{ }^{(\kappa)}, \cdots, m_{r_{\kappa}}{ }^{(\kappa)}$. In like manner, if $G(\xi, \eta)=0$ be the equation of our curve transformed to tangential coördinates, we shall have a number $r_{\kappa}$ of cycles corresponding to a value $\xi=\alpha_{\kappa}$ of orders $\bar{v}_{1}{ }^{(\kappa)}, \cdots, \bar{v}_{r_{\kappa}}{ }^{(\kappa)}$, respectively, and for the function $G_{\eta}{ }^{\prime}(\xi, \eta)$ a set of lowest exponents $\bar{m}_{1}{ }^{(\kappa)}, \cdots, \bar{m}_{\bar{r}_{\kappa}}{ }^{(\kappa)}$. Plücker's formulæ may then be replaced by the three formulæ

$$
\begin{gathered}
\sum_{\kappa} \sum_{s=1}^{r_{\kappa}} m_{s}{ }^{(\kappa)} v_{s}(\kappa)=0, \quad \sum_{\kappa} \sum_{s=1}^{\bar{r}_{\kappa}} \bar{m}_{s}{ }^{(\kappa)} \bar{v}_{s}^{(\kappa)}=0, \\
\sum_{\kappa} \sum_{s=1}^{r_{k}}\left(v_{s}{ }^{(\kappa)}-1\right)-\sum_{\kappa} \sum_{s=1}^{\bar{r}_{\kappa}}\left(\bar{v}_{s}^{(\kappa)}-1\right)=2(n-\bar{n}),
\end{gathered}
$$

where the respective summations are extended to all values of the variable $z$, or to all values of $\xi$ as the case may be, the values $z=\infty$ and $\xi=\infty$ included. The first two formulæ follow from the theorem that a rational function has as many zeros as infinities, the last formula from the equality of the genus of the equation $F(z, u)=0$ and that of the transformed equation $G(\xi, \eta)=0$.

The three formulæ in the above form appear of themselves in a general algebraic theory of the algebraic functions which the author has developed and which he expects to publish shortly. They present themselvès in the theory of the algebraic curves possessing higher singularities under those circumstances where in the ordinary theory of algebraic curves Plücker's formulæ occur, and furthermore in such a way that the customary question in regard to the combination of simple singularities to which a higher singularity is equivalent becomes superfluous, at least from an algebraic standpoint.

In Professor Newson's paper it is shown that the value $x_{i}$ in the product of two linear substitutions $T$ and $T_{1}$ in $n$ 
variables is proportional to the determinant formed by bordering the determinant $T^{-1}$, the inverse of $T$, vertically by the variables of $T$, and horizontally by the coefficients of the $i$ th equation in $T_{1}$. The paper will be published in an early number of the Annals of Mathematics.

Burnside determined the groups of order $p^{m}, p$ being any prime number, which contain a cyclic invariant subgroup of order $p^{m-2}$. In a recent article published in the Transactions of this Society, Professor Miller determined those groups of order $p^{m}$ which contain the abelian group of type $(m-2,1)$. His present paper is devoted to a study of the remaining groups of order $p^{m}$ which contain operators of order $p^{m-2}$. It is proved that there are just two such groups when $p>2$ and $m>5$, while there are five when $p=2$ and $m>5$. In the first case $(p>2, m>5)$ there are therefore 15 groups which contain operators of order $p^{m-2}$-four are abelian, five others contain an invariant cyclic subgroup of order $p^{m-2}$, while the remaining six do not have either of these properties. Each of these 15 groups is conformal with some abelian group. In the second case $(p=2, m>5)$ there are 33 groups which contain operators of order $2^{m-2}$-four of these are abelian, eighteen others contain an invariant cyclic subgroup of order $2^{m-2}$, while eleven do not possess either of these properties. These eleven are composed of $G_{8}, G_{9}, G_{12}, G_{13}, G_{14}$, and $G_{17}$ of the article mentioned and the five groups of the present article. The full paper will be offered for publication to the Transactions.

In recent papers in the Transactions of this Society and in the Proceedings of the London Mathematical Society, Professor Dickson investigated several classes of linear groups in an arbitrary realm of rationality. The earlier methods applicable to finite fields were replaced by developments involving only the rational processes of algebra. Among the groups considered are those defined by quadratic invariants. The latter, for finite fields of modulus $p>2$, may be given the canonical forms

$$
\begin{array}{ll}
\xi_{1}{ }^{2}+\cdots+\xi_{m-1}{ }^{2}+\xi_{m}{ }^{2} & (m \text { even or odd }), \\
\xi_{1}{ }^{2}+\cdots+\xi_{m-1}{ }^{2}+\nu \xi_{m}^{2} & (m \text { even }),
\end{array}
$$

where is a not-square. As another set of canonical forms, for finite fields, we may take

$$
f \equiv \xi_{1} \eta_{1}+\cdots+\xi_{m} \eta_{m}, \quad \xi_{0}^{2}+f, \quad \xi_{0}^{2}-\nu \eta_{0}^{2}+f .
$$


The conditions for the invariance of $f$ under a linear transformation are bilinear in the coefficients and therefore may be treated by rational processes. Similar remarks hold for a treatment of the remaining two forms by induction from $m-1$ to $m$. The second set of canonical forms defines groups in an arbitrary realm whose structures were determined in the papers cited. Moreover, the second set proves more advantageous than the first set in the question of the representation of the linear groups as transitive substitution groups. The first set was employed in the American Journal of Mathematics, October, 1901. For the second set, the investigation becomes simpler and the results appear in a simpler and more natural classification.

Not only for these groups, but for other classes, it appears that the simplest methods are those applicable to an arbitrary realm, viz., methods which depend essentially upon rational operations only.

Thomas F. Holgate, Secretary of the Section.

\section{VECTOR ANALYSIS.}

Vector Analysis. A text-book for the use of students of mathematics and physics, founded upon the lectures of $J$. Willard GibBs, Ph.D., LL.D., Professor of Mathematical Physics in Yale University. By Edwin Bidwell Wilson, Ph.D., Instructor in Mathematics in Yale University. (Yale Bicentennial Publication.) New York, Charles Scribner's Sons, 1901. 8vo. $\quad x x+436$ pp.

IT is well known that Professor Gibbs's " Elements of vector analysis," a pamphlet of 83 pages, printed in 1881-84 for the use of his students, although not published for general circulation, attracted somewhat wide attention. Thus, in particular, Mr. Oliver Heaviside adopted Professor Gibbs's system with but slight modifications and expounded it very fully in his "Electromagnetic Theory" (1893); this again formed the basis, to a large extent, of Professor Föppl's " Einführung in die Maxwell'sche Theorie der Elektricität, mit einem einleitenden Abschnitte über das Rechnen mit Vectorgrössen in der Physik" (189k). But vector analysis, as conceived by Professor Gibbs, is not merely a method 